\title{
Mitteilung
}

\section{Neue Mitglieder in die Leitlinienkommission der AWMF berufen}

Wolfgang Müller ${ }^{1^{*}}$

${ }^{1}$ AWMF, Düsseldorf

GMS Mitteilungen aus der AWMF 2007;4:Doc09

\section{Text}

Das Präsidium der AWMF hat bei seiner letzten Sitzung beschlossen, die Ständige Kommission Leitlinien der AWMF um vier persönliche Mitglieder zu erweitern. Für die Dauer von 3 Jahren wurden auf Grund ihrer Erfahrungen und Verdienste um die Leitlinienentwicklung berufen:

- Monika Lelgemann MSc. (Klin. Epi.)

Institut für Gesundheits- und Medizinrecht (IGMR), FB Rechtswissenschaft, Universität Bremen

- Prof. Dr. med. Berthold Rzany Sc.M.

Division of Evidence Based Medicine (dEBM), Klinik für Dermatologie der Charité Berlin

- PD Dr. Karsten Schwerdtfeger

Neurochirurgische Klinik, Universitätskliniken des Saarlandes Homburg/Saar

- PD Dr. Helmut Sitter

Institut für Theoretische Chirurgie, Philipps-Universität Marburg

Die Ständige Kommission Leitlinien der AWMF hatte bereits auf ihrer letzten Sitzung beschlossen, diese vier Personen auf Grund ihrer Fortbildung und praktischen Erfahrungen in der Entwicklung von Leitlinien für die nächsten drei Jahre zu offiziellen AWMF-Leitlinien-Beratern zu ernennen. Eine Verlängerung ist möglich. 